\title{
Characterization of the motility of maturing rat spermatozoa by computer-aided objective measurement
}

\author{
C. H. Yeung, G. Oberländer and T. G. Cooper
}

Institute of Reproductive Medicine of the University, Steinfurter Straße 107, D-4400 Münster, Germany

\begin{abstract}
Summary. A computer-aided sperm analysis system was optimized for objective assessment of the movement characteristics of mature and immature rat spermatozoa by testing different settings. Measurements of straight line velocity of individual motile cells were validated by manual tracking with a digitizer. Better agreement between the two methods and better performance in distinguishing between mature and immature spermatozoa was obtained by reducing the tracking rate to increase the time of analysis. However, numbers of motile and immotile cells could not be determined accurately. Manual counting of videotaped images revealed no significant differences in percentage motility of spermatozoa from five epididymal regions. Caput spermatozoa were characterized by low straight-line (VSL) and averaged-path (VAP) velocities and low path straightness (STR), whereas mature cells displayed high VSL, VAP and STR. An increase in curvilinear velocity on maturation was less obvious. Spermatozoa in the proximal corpus epididymidis were heterogeneous in their acquisition of motility maturation and the uniformity of movement pattern achieved in the distal corpus and proximal cauda regions tended to decrease again in the distal cauda epididymidis. Such objective measurements of motility patterns will facilitate studies on the regulation of motility development upon sperm maturation.
\end{abstract}

Keywords: epididymal spermatozoa; motility maturation; sperm analysis; computer-aid; rat

\section{Introduction}

The motility of spermatozoa changes as they pass through the epididymis. The motility pattern of spermatozoa obtained from the caput and cauda epididymidis of rats has been described as circling in the former and progressive in the latter (Blandau \& Rumery, 1964; Wyker \& Howards, 1977). The circling motion has been ascribed to a greater rigidity in the mid-piece that does not partake in flagellar bending, and a lack of rotation of the immature cell (Fray et al., 1972; Burgos \& Tovar, 1974). These differences in motility of caput and cauda spermatozoa were considered by Blandau \& Rumery (1964) to be responsible for their differences in fertilizing capacity in vivo, since fewer caput spermatozoa were found in the oviducts after uterine insemination. This was confirmed by Gaddum-Rosse (1981), who examined the oviductal aspect of the uterotubal junction from tissues freshly excised after mating or insemination and found that only forward progressing spermatozoa could negotiate it.

Attempts to quantify the changes in movement of rat spermatozoa upon maturation include subjective grading, which suggested a stepwise increase from the caput through the corpus to the cauda, measuring migration in capillary tubes, which indicated less marked increases in distance travelled (Turners \& Giles, 1981), and computation of the frequency of area change caused by sperm tail flagellation, which failed to distinguish between spermatozoa from the distal caput and the more distal epididymal regions (Hinton et al., 1979). In contrast to the detection of the 
movement of spermatozoa in a sample as a whole, computer-aided sperm analysis (CASA) has been developed in the past decade to measure the movement of individual cells (see Boyers $e t$ al., 1989) and has been increasingly applied to measure sperm movement objectively, mainly in clinical studies (see Knuth et al., 1989; Boyers et al., 1989). With the existing commercial CASA systems, errors in the identification of motile and immotile sperm cells have been recognized (human: Knuth \& Nieschlag, 1988; Neuwinger et al., 1990; monkey: Yeung et al., 1989) and the settings of the various CASA parameters can alter results of analysis (human: Knuth et al., 1987; Vantman et al., 1988; rat: Toth et al., 1989). However, few studies have evaluated the automatic tracking of individual motile spermatozoa (human: DeGeyter et al., 1988; Mack et al., 1988; Olds-Clarke et al., 1990; monkey: Yeung et al., 1989) which is fundamental to the generation of meaningful data. The major concern here is the correct tracking of the same motile cell from frame to frame, since once tracked correctly, the precision of the measurement of various parameters obtained using known standards is within $2 \%$ (Douglas-Hamilton et al., 1990) and higher than any manual tracking.

CASA has been used to analyse the motility parameters of rat spermatozoa, but to date only mature spermatozoa have been examined (Working \& Hurtt, 1987; Toth et al., 1989, 1991; Slott et al., 1990; Klinefelter et al., 1990, 1991). In the present study the Hamilton-Thorn system (HTM, Hamilton Thorn Research Inc., Beverly, Mass) is validated for rat spermatozoa and used to detect changes as they mature in the epididymis. The measurement of straight-line velocity for rat cauda spermatozoa by the HTM has previously been validated by measuring images on the video monitor manually and comparing mean values (Slott et al., 1990). By checking the measurement of each individual motile cell, the present study shows that with the optimal setting the HTM system could track correctly and distinguish the movement patterns of immature and mature motile rat spermatozoa.

\section{Materials and Methods}

\section{Animals and sampling of spermatozoa}

Adult Sprague-Dawley rats $(350-420 \mathrm{~g})$ were kept on a schedule of $12 \mathrm{~h}$ light: $12 \mathrm{~h}$ dark and had access to rat chow and water ad libitum. Experiments were performed in accordance with the German animal protection regulations. Spermatozoa were obtained either from epididymal tubules incubated in vitro or from various regions of the epididymis of urethane anaesthetized rats $(0.6 \mathrm{~g}$ ethyl carbamate per $100 \mathrm{~g}$ body weight). Lengths of tubule from the distal cauda epididymidis were isolated by ligatures $(8 / 0$ Barraquer silk) and were immersed in a medium similar to that described by Meizel et al. (1980; $123 \mathrm{mmol} \mathrm{NaCl}{ }^{-1}, 4 \mathrm{mmol} \mathrm{KCl}{ }^{-1}, 2 \mathrm{mmol} \mathrm{CaCl}_{2} \mathrm{l}^{-1}, 0.4 \mathrm{mmol} \mathrm{MgSO}_{4} \mathrm{l}^{-1}$, $0.3 \mathrm{mmol} \mathrm{Na}_{2} \mathrm{HPO}_{4} 1^{-1}, 25 \mathrm{mmol} \mathrm{NaHCO} 1^{-1}, 5 \mathrm{mmol}$ glucose $1^{-1}, 12.5 \mathrm{mmol}$ sodium lactate $1^{-1}$ and $0.5 \mathrm{mmol}$ pyruvate $\mathrm{l}^{-1}, 8 \mu \mathrm{g}$ phenol red $\mathrm{ml}^{-1}, 4 \mathrm{mg}$ bovine serum albumin $\mathrm{ml}^{-1}$, with a $\mathrm{pH}$ of 7.4 and osmolality of $310 \mathrm{mOsm}$ $\mathrm{kg}^{-1}$ ) in 24-well plates held at $34^{\circ} \mathrm{C}$ in a humid atmosphere with $5 \% \mathrm{CO}_{2}$ in air. At each period (up to $4 \mathrm{~h}$ ) short segments of the tubule were cut off and transferred to the end of a plastic cuvette spatula onto which the contents were extruded and the remaining tubule was removed. Luminal contents were dispersed in about $0.1 \mathrm{ml}$ medium.

Anaesthetized animals were kept on a warmed $\left(37^{\circ} \mathrm{C}\right)$ table and the testes and epididymides were exposed through scrotal incisions and warmed $\left(33^{\circ} \mathrm{C}\right)$ in baths of mineral oil. Tubules were punctured in the caput (region 2), proximal corpus (mid-way between regions 3 and 4), distal corpus (mid-way between regions 4 and 5), proximal cauda (midway between regions 5 and 6 ) and distal cauda epididymidis (region 7, numbered regions as cited in Hinton et al., 1979). Luminal contents were collected within $2 \mathrm{~h}$ of anaesthesia from exposed epididymal tubules cut in areas free of blood vessels, on the end of small, drawn-out glass rods. The order of collecting spermatozoa from different regions was rotated between experiments to randomize effects of anaesthesia on the in vivo maintenance of the spermatozoa. Luminal contents were dispersed by gentle swirling in about $0.1 \mathrm{ml}$ of the medium above.

All sperm suspensions were further diluted in the same medium when necessary to achieve a concentration that was compatible with optimal tracking by the HTM (about $2 \times 10^{5} \mathrm{ml}^{-1}$ ) and a sample was videotaped for 5 min for analysis (see below) starting about 2 min after the collection of spermatozoa.

\section{Videorecording of sperm preparation}

Sperm suspensions ( $21.8 \mu \mathrm{l}$, delivered by positive displacement pipette, Gilson Microman, France) were placed on grease-free, siliconized slides (Sigmacote: Sigma Chemie) prewarmed on a heated platform $\left(35^{\circ} \mathrm{C}\right)$ and covered by $21 \times 26 \mathrm{~mm}^{2}$ coverslips to achieve a calculated chamber depth of $40 \mu \mathrm{m}$, as there should be no appreciable spreading 
of fluid beyond the edges of the coverslip when siliconized slides were used. Slides were transferred to a heated stage (equilibrated chamber temperature $31^{\circ} \mathrm{C}$ ) of an Olympus BH2 microscope. An objective $(\times 4)$ was used with a $\times 40$ phase ring to produce pseudo-dark-field optics. Videorecording was made at 50 frame $\mathrm{s}^{-1}$ using a photo-ocular $(3.3 \times)$, a camera (Sony DXC 1850P, Tokyo, Japan) and a videorecorder (Panasonic S-VHS 100EG: Mitsushita Electric Industrial Co. Ltd, Japan) interfaced with a digital timer (VTG-33: ForA Co. Ltd, Japan), at microscope light setting 6. Each chamber was systematically scanned for $5 \mathrm{~min}$ and each field for about $10 \mathrm{~s}$.

\section{Analysis of sperm kinematics}

Percentage of motile cells. The percentage of motile spermatozoa in each sample was obtained from viewing the video-replay. The number of motile spermatozoa in each field was counted manually at one time and the total number of spermatozoa was obtained with the frame frozen at that time. A total of 200-300 spermatozoa in each sample was counted.

HTM parameters. The CASA system Hamilton-Thorn HTM 2030 (version 6.4xE: Hamilton Thorn Research, Inc., Beverly, MA, USA) was used to assess sperm kinematics. After optimization (see below), settings of the HTM used were: minimum contrast 10 ; minimum size 12 ; low/high gate for both size and intensity $0.4 / 2 \cdot 5$; no threshold for curvilinear velocity was set (all motile spermatozoa analysed had average path velocities $>5 \mu \mathrm{m} \mathrm{s}^{-1}$ ); magnification setting 0.52 ; analysis rate set at 7 frame $\mathrm{s}^{-1}$ (total 20 frames analysed over $3.04 \mathrm{~s}$; i.e. actual tracking rate 6.57 frame $\mathrm{s}^{-1}$ ); default pixel size 20; default pixel intensity 500 .

Data handling. At each analysis, the playback picture was scrutinized for occasional errors including artefacts generated by the digital timer display, flagellating spermatozoa stuck to the slide and drifting cells. These were deleted using the edit track function and the remaining sperm track data were transferred and stored on disc. For each sample, 100-200 motile spermatozoa or all fields recorded were analysed with the HTM. All sperm tracks with $<6$ track points were deleted before data processing and analysis using commercial spreadsheet (Lotus Development Corp., Cambridge, MA, USA) and statistical (Statgrafics, Rocksville, MD, USA) packages.

Motion parameters measured. With the optical and video systems used, a rat spermatozoon appeared bright against a dark background, with the head and two-thirds of the tail visible. The CASA system detected the bright image and located the centroid of this luminosity, which was about half way along the mid-piece of the spermatozoon. The HTM tracked the movement of the centroid from one video frame to the next. Kinematic parameters measured were abbreviated according to Boyers et al. (1989) and defined as: VCL, curvilinear velocity (termed 'track speed' by HTM): the time-averaged velocity obtained from distances between two consecutive track points; VSL, straight-line velocity (termed 'progressive velocity' by HTM): the velocity obtained from the distance between the first and the last track points; VAP, average-path velocity: the time-averaged velocity obtained from smoothing the original path which joined up consecutive track points, using a 5-point running average; ALH, amplitude of lateral displacement of the centroid: the width of centroid oscillation measured as twice the maximum value of the distance of any point on the original track from the corresponding 5-point average (it should be noted that by definition this is double the ALH values of most other CASA systems); LIN, linearity: (VSL/VCL) $\times 100 \%$; STR, straightness (termed 'linearity index' by HTM): (VSL/VAP) $\times 100 \%$. The mean coefficients of variation obtained from five samples when each sample was measured twice using the HTM were: VCL $1.3 \%$, VSL $3.5 \%$, VAP $1.5 \%$, STR $3.6 \%$ and ALH $5.9 \%$.

\section{Optimization of settings for HTM performance}

Changing default settings. Video-recordings of distal cauda epididymal spermatozoa prepared from tubules held in vitro were analysed using the HTM at 25 frame $\mathrm{s}^{-1}$. The same field at the same time point of recording ( \pm 2 videoframes, i.e. $\pm 0.08 \mathrm{~s}$ variation) was analysed at different settings and was scrutinized using the 'play back' function of the HTM to check whether motile spermatozoa (indicated by a red dot on the HTM monitor) and immotile spermatozoa (indicated by a green dot) were correctly identified. Preliminary trials indicated that minimum contrast $<10$ or minimum size $<11$ or low intensity gate $<0.3$ led to a tendency to identify a single cell as two; whereas parameter settings of $>12,>14$ or $>0.4$, respectively, led to non-detection of motile cells. On the basis of these observations, eight different combinations of settings (Table 1) were chosen to analyse 11 fields containing 73 motile and 78 immotile spermatozoa. From these eight settings, the better four were used to analyse more spermatozoa.

Validation of VSL measurements by manual assessment. Straight-line velocities obtained from the HTM for each individual motile sperm cell were compared with values obtained by tracking manually the position of the sperm head using a digitizing morphometry system (MOP-Videoplan: Kontron Electronic Group, Zeiss, Oberkochem, Germany) to measure the distance moved by the same sperm cells during the same time interval. For each field analysed by the HTM the positions of motile spermatozoa on the HTM monitor were marked and numbered on an overlying acetate sheet. This was used to identify the corresponding motile cell on the Kontron monitor. These Kontron measurements were used as the basis for comparison to determine which HTM setting was optimum. For the initial validation, video-fields containing any spermatozoa swimming across each other were not used for analysis.

Changing tracking. Video-recordings of spermatozoa from the caput and the distal corpus epididymidis were analysed using the best HTM setting (no. 4 in Table 1, see Results) first at 25 and then at 7 frame $\mathrm{s}^{-1}$. VSL data measured at 7 frame $^{-1}$ were compared with those obtained by manual tracking as described above. The coefficients 
of variation in VSL obtained by manual tracking were 11.7 and $1.7 \%$ for caput and distal corpus spermatozoa (duplicate measurements of 20 cells each), respectively.

\section{Statistical analysis}

For the optimization and evaluation of the HTM analysis of variance, linear regression and analysis of the differences of paired data were performed. For the effect of maturation on sperm kinematics, data were analysed using Friedman's rank test on the median value of each sample.

\section{Results}

\section{Optimization of the HTM}

\section{Different settings at 25 frames $\mathrm{s}^{-1}$}

With the eight HTM settings tested initially (Table 1) most, though not all, of the motile cells were detected as single cells. However, some motile spermatozoa were counted more than once (owing to the splitting of tracks, see later section). The recognition of immotile spermatozoa was sometimes erroneous in that the number counted from viewing the monitor showed no significant correlation with the number in the same field analysed by the HTM (data not shown). In this study, therefore, sperm counts from the HTM were not used and percentage motility was estimated from manual counts.

\section{Validation of VSL from mature sperm tracks at 25 frames $\mathrm{s}^{-1}$}

Judged from comparisons of HT values with individual straight-line velocity data obtained by manually tracking the motile cauda spermatozoa, the minimum size setting of 12 appeared to be better than the higher or lower setting (Table 1), in terms of coefficients of correlation and paired differences. Although the four better settings produced similar measurements to those obtained using the Kontron digitizer as shown in Table 1, the distribution pattern of the data from setting no. 4 (Fig. 1a) fitted better to that of manual tracking than distribution curves from the other settings, which appeared bimodal (Fig. 1b). With all settings tested, there were always some (a few per cent of the total) motile cells demonstrating splitting of the swim-path of a single sperm cell into two or more tracks (experiment 2 in Table 1), although for this part of the validation sperm paths crossing with one another were excluded from analysis. Splitting of tracks was the result of either a collision of the motile cell with an immotile cell, or a drastic change of the shape of the luminous image from one frame to the next such that the centroid was outside the individual detection area (this setting was not adjustable in the present HTM version) and was considered as a different cell. Less frequently this was due to the detection of the cell as two images owing to bending of the midpiece perpendicular to the plane of focus which resulted in an uneven brightness of the image as a whole. However, this splitting of tracks occurred randomly in that the cells with split tracks did not reflect any subpopulation of the motile spermatozoa (Fig. 1a). As a consequence, inclusion of data from these split tracks did not bias the assessment of the whole population.

\section{Changing tracking rate to distinguish motility patterns}

Compared with motile spermatozoa obtained from the more distal epididymal regions, which produced relatively straight paths, those from the caput epididymidis had more rigid and curved mid-pieces (in P bends as defined by Woolley (1977)), rotated along their axes less frequently and exhibited circular or tortuous swim-paths. Although this difference in movement patterns was easily discernible by observing the movement on the video-monitor, analysis at 25 frame $\mathrm{s}^{-1}$ for 20 frames using the HTM with the optimal setting failed to manifest such differences. Kinematic parameters of distal corpus spermatozoa were similar or only slightly higher in values than caput spermatozoa (Table 2). However, significant differences became clearer when the samples were 


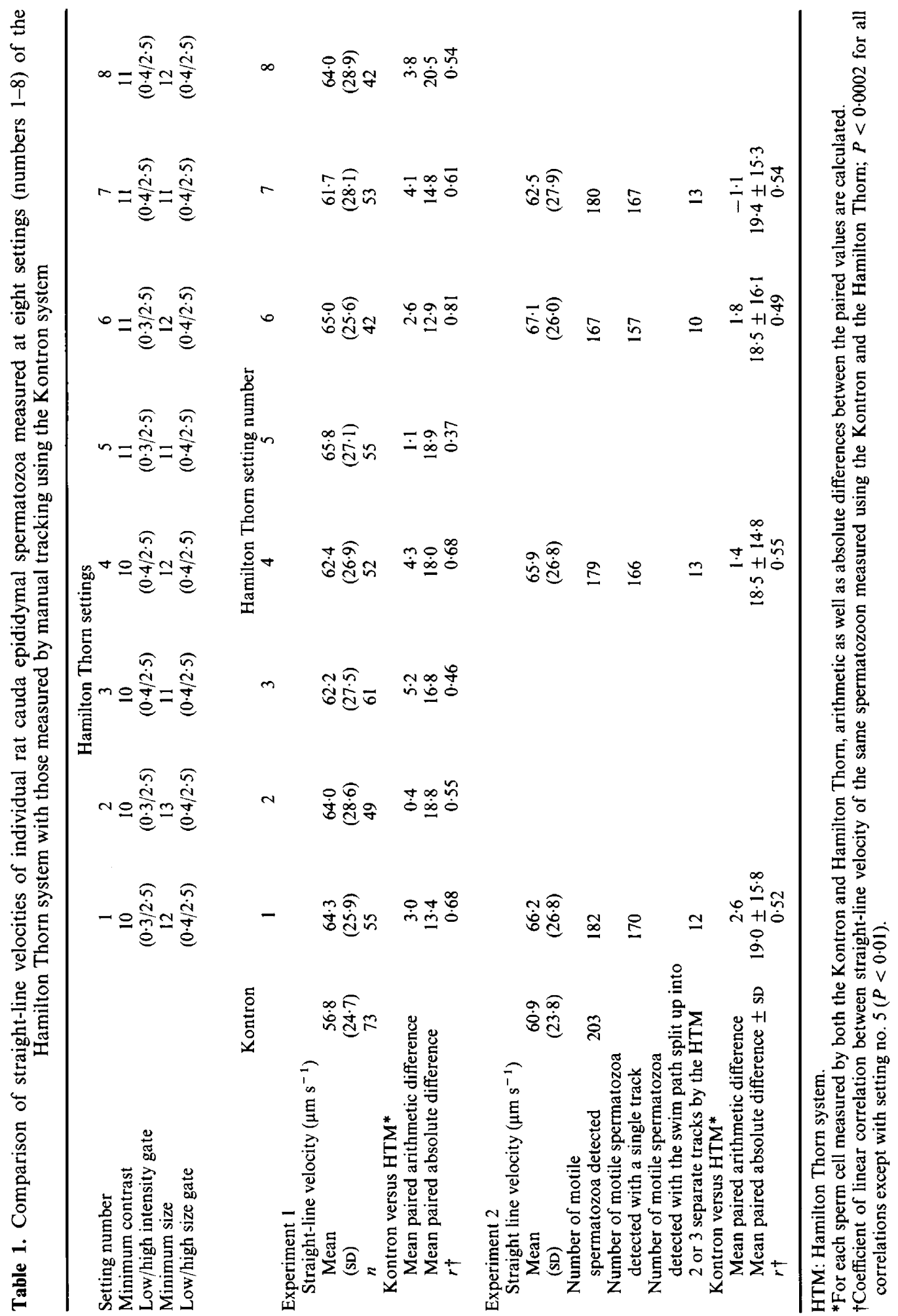




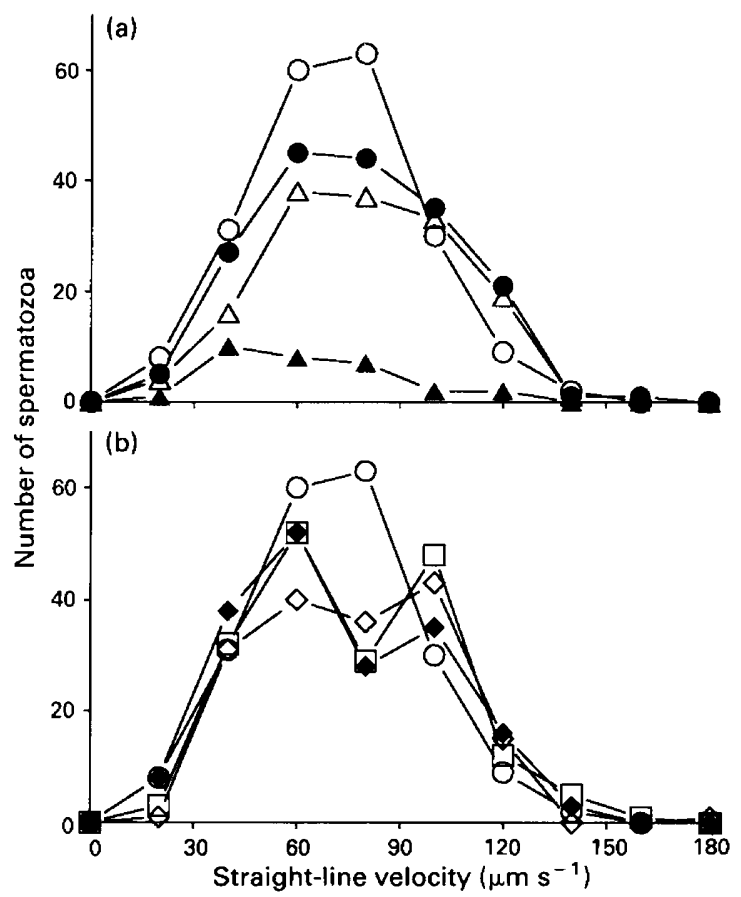

Fig. 1. Comparison of distribution curves of straight-line velocities of rat cauda spermatozoa measured using different Hamilton Thorn settings (see Table 1): (a) setting 4: (O) all tracks; $(\triangle)$ single tracks; $(\Delta)$ split tracks; $(O)$ using the Kontron digitizer with manual tracking; (b): all tracks; $(\square)$ setting $1 ;(\diamond)$ setting $6 ; \diamond)$ setting 7 and $(O)$ using the Kontron digitizer with manual tracking.

reanalysed for 20 frames at a different tracking rate of 7 frame $\mathrm{s}^{-1}$ (real rate 6.57 frame $\mathrm{s}^{-1}$ ), which in effect increased the total tracking time interval from 0.76 to $3.04 \mathrm{~s}$ (Fig. 2). As a result all velocity values decreased more drastically for caput than distal corpus spermatozoa, such that VSL and STR values of the mature cells were 140 and $70 \%$, respectively, higher than those of the immature cells (Table 2).

\section{Validation of mature and immature sperm tracks at 7 frames $s^{-1}$ over $3.04 \mathrm{~s}$}

In a preliminary experiment, recorded motile spermatozoa obtained from the caput and the distal corpus epididymidis were analysed both by manual tracking and by the HTM with the longer tracking interval. All motile spermatozoa were tracked by the HTM (Table 3). Paired data of straight-line velocities of individual cells measured by the two methods showed little differences (Table 3) and good correlations for both caput and distal corpus spermatozoa (Fig. 3a, b; $r=0.64$ and 0.92 , respectively, $P<0.001$ for both regression lines) with closely fitting distribution curves (Fig. 4). This setting of lower tracking rate was therefore used in further experiments.

In the above validation, spermatozoa swimming across one another were excluded from analysis. This, however, is difficult to avoid with sperm concentrations enabling enough motile spermatozoa to be recorded in $5 \mathrm{~min}$. When crossing occurred, the tracks from single cells were often split and sometimes merged with one another. The effect of crossing on the kinematic assessment of the whole population was checked by analysing three samples with different mean velocities and frequent crossing of sperm paths. Results obtained before and after deletion of tracks arising from crossing spermatozoa from the data files were compared. Inclusion of crossings in the analysis did not affect the result of the whole samples (Table 4). 
Table 2. Effect of changing the interval and the rate of tracking motile cells by the Hamilton Thorn system on the measurement of motility parameters of spermatozoa from the caput and distal corpus epididymidis from one rat epididymis

\begin{tabular}{|c|c|c|c|}
\hline & $\begin{array}{l}\text { Caput } \\
\text { (20 fields) }\end{array}$ & $\begin{array}{l}\text { Distal corpus } \\
\text { (29 fields) }\end{array}$ & $\begin{array}{l}\text { Distal corpus } \\
\text { (\% caput value) }\end{array}$ \\
\hline \multicolumn{4}{|l|}{$\begin{array}{l}\text { Twenty frames analysed over } 0.76 \mathrm{~s} \\
\text { (set at } 25 \text { frame } \mathrm{s}^{-1} \text { ) }\end{array}$} \\
\hline Number of tracks & 154 & 172 & \\
\hline Straight-line velocity $\left(\mu \mathrm{m} \mathrm{s}^{-1}\right)$ & $40 \cdot 7 \pm 2 \cdot 4$ & $56.8 \pm 2.8$ & 139 \\
\hline Average path velocity $\left(\mu \mathrm{m} \mathrm{s}^{-1}\right)$ & $101 \cdot 7 \pm 3 \cdot 1$ & $111.8 \pm 3.8$ & 110 \\
\hline Curvilinear velocity $\left(\mu \mathrm{m} \mathrm{s}^{-1}\right)$ & $178 \cdot 5 \pm 5 \cdot 4$ & $208 \cdot 9 \pm 5 \cdot 1$ & 117 \\
\hline Straightness (\%) & $40 \cdot 6 \pm 1 \cdot 8$ & $52 \cdot 4 \pm 1 \cdot 8$ & 129 \\
\hline Lateral head displacement $(\mu \mathrm{m})$ & $21 \cdot 4 \pm 0.9$ & $23 \cdot 1 \pm 0.7$ & 108 \\
\hline \multicolumn{4}{|l|}{$\begin{array}{l}\text { Twenty frames analysed over } 3.04 \mathrm{~s} \\
\text { (set at } 7 \mathrm{frame} \mathrm{s}^{-1} \text { ) }\end{array}$} \\
\hline Number of tracks* & 347 & 347 & \\
\hline Straight-line velocity $\left(\mu \mathrm{m} \mathrm{s}^{-1}\right)$ & $15.9 \pm 0.5$ & $38 \cdot 0 \pm 0.9$ & 239 \\
\hline Average path velocity $\left(\mu \mathrm{m} \mathrm{s}^{-1}\right)$ & $32 \cdot 6 \pm 0 \cdot 7$ & $43.8 \pm 0.9$ & 134 \\
\hline Curvilinear velocity $\left(\mu \mathrm{m} \mathrm{s}^{-1}\right)$ & $68 \cdot 6 \pm 1 \cdot 2$ & $59 \cdot 9 \pm 1 \cdot 1$ & 87 \\
\hline Straightness $(\%)$ & $51 \cdot 0 \pm 1 \cdot 2$ & $86.2 \pm 0.9$ & 169 \\
\hline Lateral head displacement $(\mu \mathrm{m})$ & $29 \cdot 8 \pm 0 \cdot 7$ & $19 \cdot 4 \pm 0.5$ & 65 \\
\hline
\end{tabular}

Values are means \pm SEM.

*The window of detection was automatically reduced in size upon changing the setting from 7 to 25 frame $\mathrm{s}^{-1}$, resulting in fewer cells of the same video-field being analysed with the latter setting. With the latter setting the tracking of some cells was split and the split tracks had $<6$ points and were therefore deleted from computation of results.

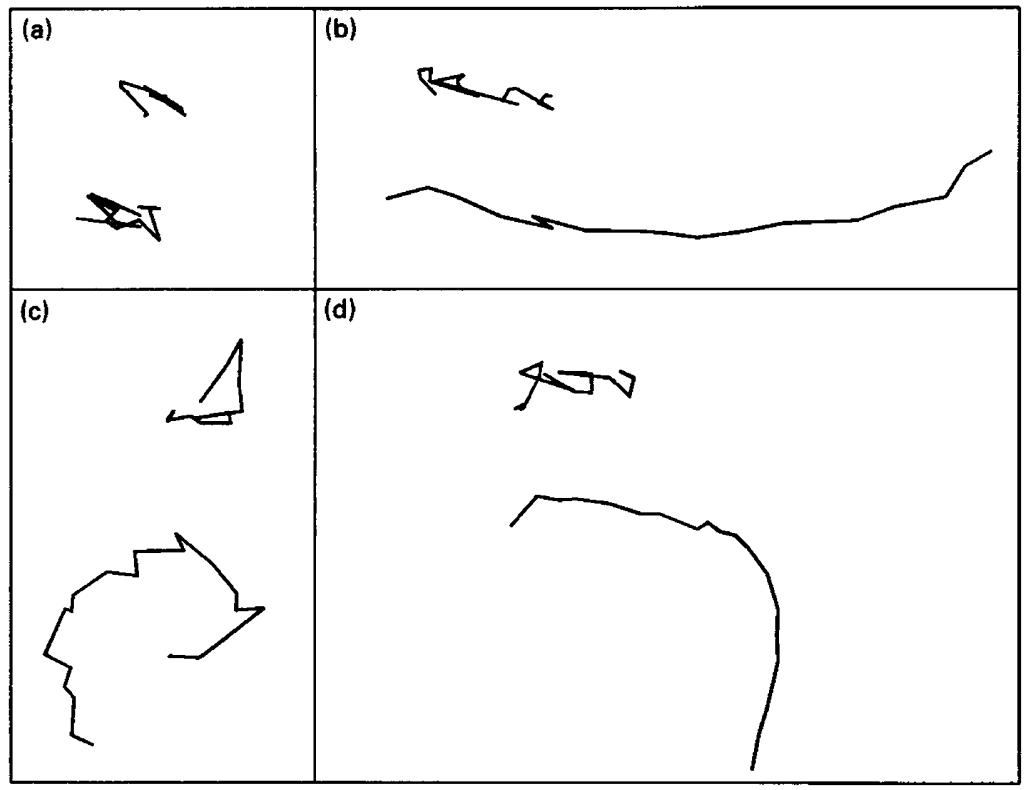

Fig. 2. Swim paths of four rat spermatozoa, two from the caput $(a, c)$ and two from the distal corpus $(b, d)$ epididymidis, each tracked for 20 frames at 25 frame $\mathrm{s}^{-1}$ (upper track) and at 6.6 frame $\mathrm{s}^{-1}$ (lower track) by the Hamilton Thorn analysing system. Note that differences among the four spermatozoa are more obvious from the tracks obtained with the lower tracking rate. 
Table 3. Comparison of paired data on straight-line velocity $\left(\mu \mathrm{m} \mathrm{s}^{-1}\right)$ of individual rat spermatozoa from the caput and distal corpus epididymidis measured by manual tracking (Kontron) and by the Hamilton Thorn system

\begin{tabular}{lrrrr}
\hline & \multicolumn{1}{c}{ Caput } & $(n)$ & Distal corpus & $(n)$ \\
\hline Kontron & $15 \cdot 8 \pm 7 \cdot 2$ & $(98)$ & $41 \cdot 2 \pm 14 \cdot 9$ & $(98)$ \\
HTM & $15 \cdot 6 \pm 7 \cdot 4$ & $(98)$ & $40 \cdot 6 \pm 15 \cdot 2$ & $(98)$ \\
Arithmetic difference* & $0 \cdot 2 \pm 6 \cdot 2$ & & $0 \cdot 5 \pm 6 \cdot 1$ & \\
Absolute difference & $4 \cdot 9 \pm 3 \cdot 8$ & & $4 \cdot 4 \pm 4 \cdot 2$ & \\
\hline
\end{tabular}

Values are means $\pm \mathrm{SD}$.

$n$ : number of motile spermatozoa analysed.

${ }^{*}$ Difference between paired data from Kontron and HTM analysis.

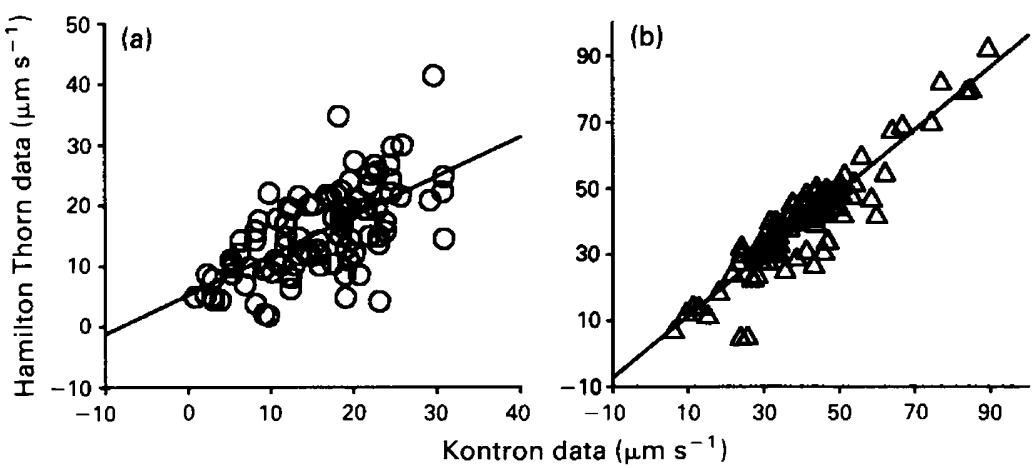

Fig. 3. Correlations of straight-line velocities of rat (a) caput and (b) distal corpus spermatozoa measured using Hamilton Thorn and Kontron digitizer (manual tracking).

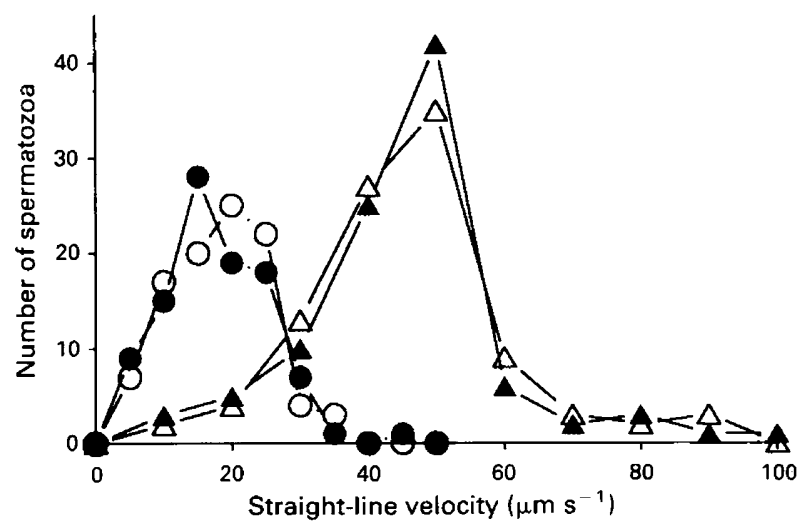

Fig. 4. Comparison of distribution curves of straight-line velocities measured using the Hamilton Thorn (closed symbols) and Kontron digitizer (manual tracking; open symbols). $(\bullet, \bigcirc)$, caput epididymidis; $(\boldsymbol{\Lambda}, \triangle)$, distal corpus epididymidis.

Effect of changes of the movement characteristics of spermatozoa upon maturation in the epididymis

\section{Percentage motility}

There was considerable variation, but no significant change, in the percentage motility of spermatozoa from all regions of the epididymis, although there was a tendency towards an increase 
Table 4. Effect of crossing swim-paths on kinematic measurements of three rat sperm samples, expressed as percentage differences $(\%$ difference $=100[x-y] /$ $y)$ of mean values obtained from data including $(x)$ and excluding $(y)$ the tracks

from crossing cells

\begin{tabular}{lccr}
\hline Sample & 1 & 2 & 3 \\
Track no. in $x$ & 228 & 159 & 191 \\
Track no. in $y$ & 132 & 119 & 132 \\
& & & \\
& & & \\
& & Difference & \\
Straight-line velocity $\left(\mu \mathrm{m} \mathrm{s}^{-1}\right)$ & $4 \cdot 4(36 \cdot 6)$ & $-0.4(15 \cdot 4)$ & $1 \cdot 3(26 \cdot 7)$ \\
Curvilinear velocity $\left(\mu \mathrm{m} \mathrm{s}^{-1}\right)$ & $-3 \cdot 5(57 \cdot 4)$ & $-2 \cdot 6(59 \cdot 2)$ & $-1 \cdot 8(66 \cdot 0)$ \\
Average path velocity $\left(\mu \mathrm{m} \mathrm{s}^{-1}\right)$ & $1 \cdot 0(40 \cdot 9)$ & $-4 \cdot 7(29 \cdot 4)$ & $-2 \cdot 7(37 \cdot 5)$ \\
Straightness $(\%)$ & $5 \cdot 2(64 \cdot 4)$ & $4.0(55 \cdot 2)$ & $3 \cdot 0(70 \cdot 2)$ \\
Lateral head displacement $(\mu \mathrm{m})$ & $-9 \cdot 1(20 \cdot 7)$ & $-4 \cdot 3(25 \cdot 8)$ & $-0 \cdot 2(26 \cdot 6)$ \\
\hline
\end{tabular}

Mean values for $y$ in parentheses.

from the caput to the cauda epididymidis, reaching a maximum of about $75 \%$ in the proximal cauda (Fig. 5a).

\section{Motion parameters}

When data from all six experiments were summarized, velocity parameters showed a marked increase upon maturation, with curvilinear velocities reaching a peak (about $80 \mu \mathrm{m} \mathrm{s}^{-1}$, Fig. 5b) in the distal corpus epididymidis, whereas the straight-line and averaged path velocities reached plateaux (VAP about 55 and VSL about $50 \mu \mathrm{m} \mathrm{s}^{-1}$, Fig. $5 \mathrm{c}, \mathrm{d}$ ) in the proximal cauda epididymidis. Straightness of the swim-path increased to a maximum in the proximal cauda epididymidis where spermatozoa attained the smallest lateral head displacement (Fig. 5e, f). However, a decrease in ALH was significant only between spermatozoa from the distal corpus and proximal cauda regions (Fig. 5f).

Distribution of motion parameters. Distribution curves of data from individual spermatozoa showed that there was only a slight shift in VCL towards higher values as spermatozoa matured, with the population being most heterogeneous in the proximal corpus and most homogeneous in the proximal cauda epididymidis (Fig. 6a,d). This shift upon maturation was more obvious with VAP distributions (not shown). Caput spermatozoa constituted one population with a low VSL and spermatozoa from the distal corpus and proximal cauda epididymides constituted another with a higher VSL, whereas both populations were found in the proximal corpus epididymidis (Fig. $6 \mathrm{~b}, \mathrm{e})$. The population became heterogeneous in the distal cauda. Distribution of the straightness of swim paths shifted to the right from the caput until it became almost uniform in the proximal and distal cauda epididymidis (Fig. 6c, f). Such patterns of transformation in these kinematic parameters were observed in all animals examined, some being more and others less obvious than those from the epididymis illustrated (chosen for the similarly high number of motile cells analysed in each region).

\section{Discussion}

Computer-aided sperm motion analysis normally takes advantage of the luminosity of symmetrical sperm heads under phase contrast conditions. Problems may thus be anticipated in the identification and tracking of rodent spermatozoa with their hooked heads which are not luminous under these conditions. Although dark-field optics render the rat sperm head bright, the relatively long and thick mid-piece is also luminous. It is thus not the position of the head, but the centroid of the long image of the head plus mid-piece that was tracked by the HTM in this study. Since it is more 
(a) Percentage motility

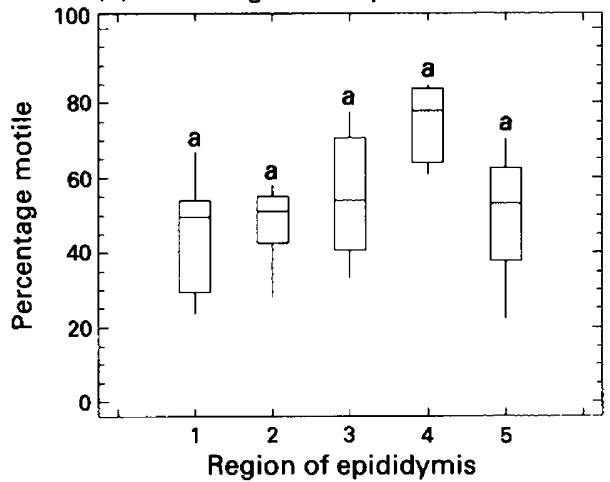

(c) Average path velocity $\left(\mu \mathrm{m} \mathrm{s}^{-1}\right)$

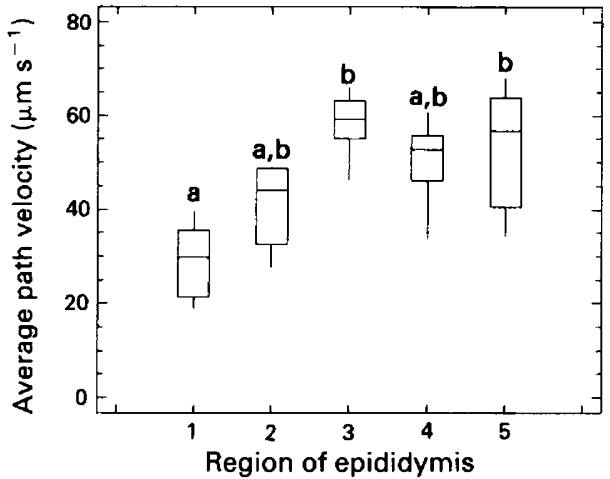

(e) Straightness (\%)

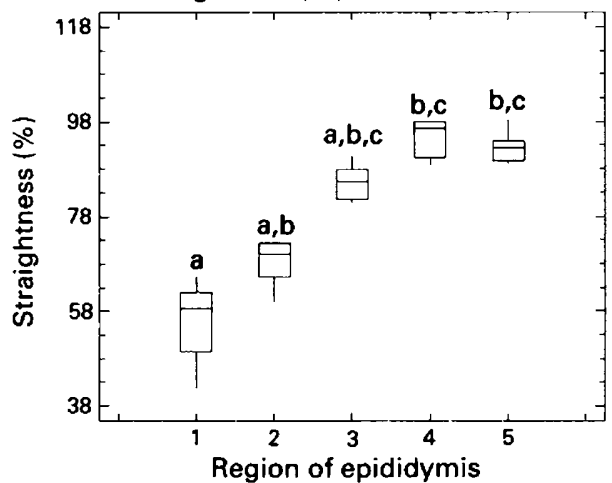

(b) Curvilinear velocity ( $\mu \mathrm{m} \mathrm{s}^{-1}$ )

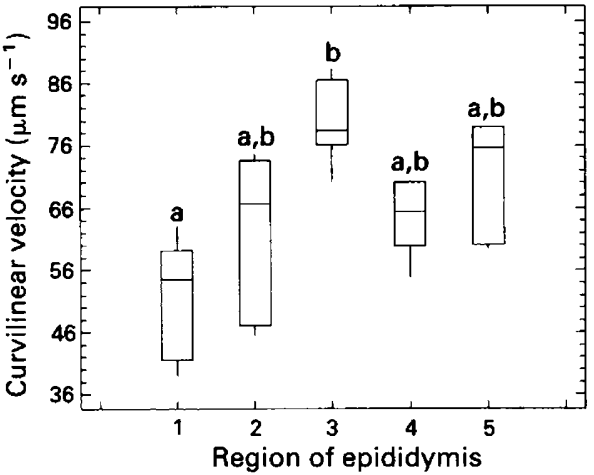

(d) Straight-line velocity ( $\left.\mu \mathrm{m} \mathrm{s}^{-1}\right)$

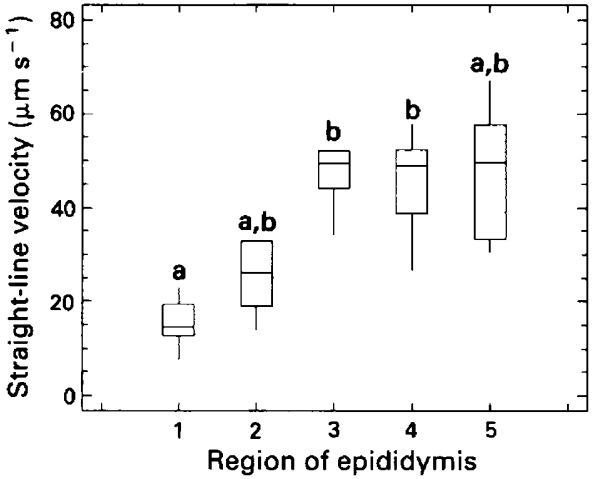

(f) Lateral head displacement $(\mu \mathrm{m})$

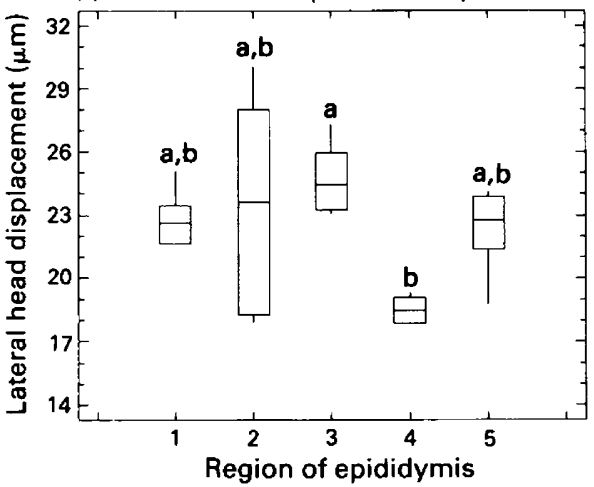

Fig. 5. Box and whisker plots of the medians of movement parameters of spermatozoa from five regions of the six rat epididymides ( 1 , caput; 2 , proximal corpus; 3 , distal corpus; 4 , proximal cauda; 5 , distal cauda epididymidis). The box indicates the quartiles and the whiskers the ranges; the line within the box is the median of six values and the individual points the outliers (outside $4 \mathrm{SD}$ of the mean calculated after excluding that outlier). For each parameter, boxes with different letters are significantly different from each other using Friedman's Rank test.

difficult for the centroid of such a long image to be stable from frame to frame, particularly when luminosity of the image may change, it is essential to validate the automatic tracking of the motile rat spermatozoa by the HTM by comparison with manual tracking. If the actual displacement of the cell were small, instability of the centroid detected by the HTM would cause a relatively large 


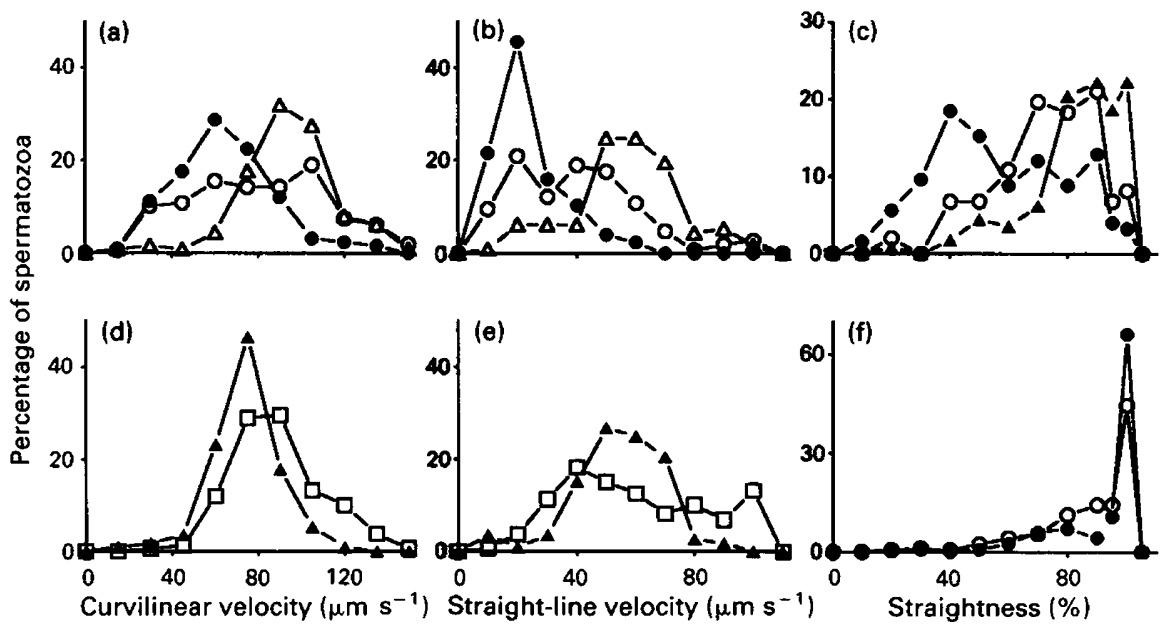

Fig. 6. Distribution curves of $(a, d)$ curvilinear velocity, $(b, e)$ straight-line velocity and $(c, f)$ straightness of swim path of spermatozoa from different regions of a rat epididymis. ( $)$ caput; $(\bigcirc)$ proximal corpus; $(\triangle)$ distal corpus; $(\Delta)$ proximal cauda; $(\square)$ distal cauda epididymidis.

deviation of the reconstructed track from the true track. In addition to this, the deviation of the path of this centroid from that of the sperm head (which was what was tracked manually) would explain the considerable absolute differences between the paired individual sperm VSL measurements obtained by the two methods when the spermatozoa were tracked at 25 frames $s^{-1}$ for 20 frames. Nevertheless, similar mean values and distributions of the whole population were obtained by the two methods. Agreement between paired individual data improved markedly when tracking was at 6.6 frame $^{-1}$ for 20 frames where the spermatozoa swam a greater distance between frames as well as for a longer total time.

The observation that the correlation between the two methods for distal corpus spermatozoa was better than for caput spermatozoa can be explained partly by the straighter paths of the more mature cell, for which the path of the head-midpiece centroid resembles that of the sperm head more closely. Caput spermatozoa also exhibited curved mid-pieces resulting in a considerable displacement of the image centroid from frame to frame due to the turning movement of the spermatozoon along its own axis, in addition to any space-gaining movement. This overestimation of the movement of the immature cell largely accounts for the poor performance of the HTM in distinguishing the movement characteristics of caput spermatozoa from the more mature spermatozoa at the tracking rate of 25 frame $s^{-1}$. Such an artefact would be considerably reduced by lowering the tracking rate to permit greater real displacement between two track points (Fig. 2). However, the problem is not the number of frame $s^{-1}$, per se, since closer approximation of the real path should be obtained with a higher tracking rate (Mortimer et al., 1988). The limitation is the duration of tracking, which can only be lengthened by decreasing the tracking rate, since the maximum number of track points is limited by the storage capacity of the CASA system (see Boyers et al., 1989).

This study shows that with the optimal settings the HTM system could track rat spermatozoa correctly and distinguish the movement patterns of immature and mature cells. However, there was splitting of tracks due to sperm collision, which is a common problem with the existing CASA systems (Boyers et al., 1989) and is difficult to avoid. This probably explains the consistent overestimation of the number of tracks by the HTM observed by Olds-Clarke et al. (1990) for human spermatozoa, which would result in erroneous counting of motile cells. Nevertheless, the present findings indicate that measurement of kinematic parameters of the spermatozoa as a population was not significantly affected by track splitting. 


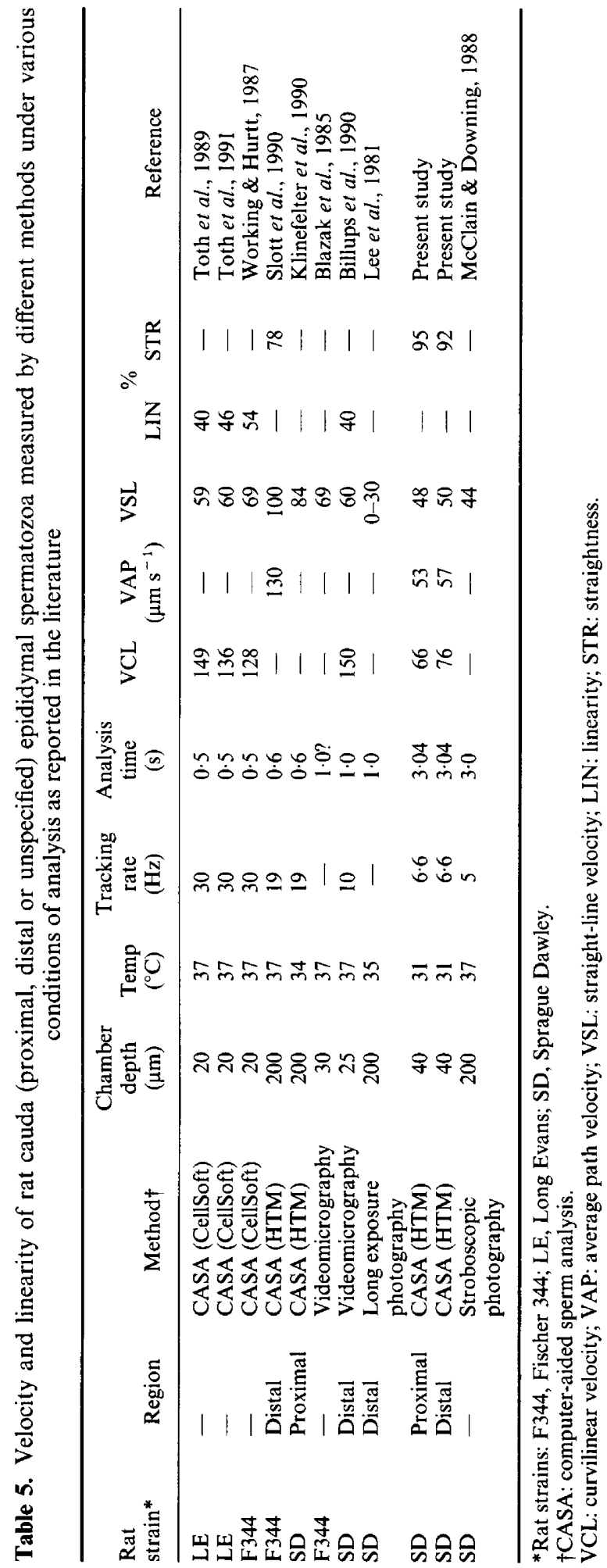


A wide range of velocities has been reported for rat cauda spermatozoa measured under various conditions (see Table 5). In addition to temperature (Rikmenspoel, 1984) and chamber depth (Ginsburg \& Armant, 1990), velocity estimates are greatly influenced by tracking rate and duration (Katz et al., 1985; Mortimer et al., 1988). This is more obvious for VCL than VSL (Table 5 ); in the present study the same sample of distal corpus spermatozoa measured at 25 frames s$^{-1}$ for $0.76 \mathrm{~s}$ showed a drastic decrease in VCL (down $67 \%$ ) when measured at 6.6 frame $\mathrm{s}^{-1}$ for $3.04 \mathrm{~s}$, whereas VSL decreased by $29 \%$. Greater values for straightness are also recorded at lower tracking rates. Great caution must therefore be taken when making comparisons of kinematic measurements between laboratories.

Examination of the distribution curves of data from individual spermatozoa revealed the development of sperm motion along the length of the epididymis in more detail. Immature spermatozoa in the caput region exhibit the characteristics of low VAP and a much lower VSL accompanied by a low linearity (STR) of the swim path. Further along the epididymal duct, spermatozoa acquired the characteristics of mature spermatozoa in terms of high VAP and an almost as high VSL due to a drastic improvement in STR. This probably resulted from a more flexible mid-piece and a symmetrically beating tail with regular rotation. The transition was completed by most spermatozoa when they reached the distal corpus or the proximal cauda epididymidis, coincident with the full acquisition of fertilizing capacity (Dyson \& Orgebin-Crist, 1973). Such marked changes, however, were accompanied by only a slight increase in VCL and an even smaller increase in percentage motility. The vigour of flagellation of distal caput spermatozoa, as measured by area change frequency, has also been reported to be similar to that of more distal regions (Hinton et al., 1979). This indicates a relatively early development in the functional capacity of the axoneme. Indeed, it has been demonstrated in the hamster that some testicular spermatozoa can beat when demembranated and activated with ATP (Mohri \& Yanagimachi, 1980) and demembranated caput spermatozoa reactivated with ATP and cyclic AMP can swim like mature cells (Yeung, 1984).

Since ageing of caput spermatozoa within the proximal epididymis does not influence the maturation to progressive motion (Burgos \& Tovar, 1974), distal epididymal secretions rather than age alone are thought to promote this change. Two compounds found in epididymal fluid can influence the motility of proximal caput spermatozoa in vitro, L-carnitine (Hinton et al., 1981) and acidic epididymal glycoprotein (AEG), the latter probably nonspecifically (Pholpramool et al., 1983). Another factor, forward motility protein (FMP), was implicated in the change in caput epididymal spermatozoan motility from a circling to a progressive motion in bulls (Acott et al., 1983) and hamsters (Kann \& Serres, 1980; Serres \& Kann, 1984) in vitro. In the hamster, retroflexion of the head brought about by such motility induction may be controlled by sulfydryl oxidation (Cornwall et al., 1986, 1988).

Movement patterns of mature sperm cells in the distal cauda epididymidis tend to diversify from the homogeneity attained in the proximal cauda, probably reflecting the heterogeneity of the ages of the stored spermatozoa. It has been suggested that mature spermatozoa aged in the epididymis have fertilizing ability inferior to younger ones in the proximal cauda, although they are still fertile as a population (Bedford, 1990).

This study was supported by the Deutsche Forschungsgemeinschaft (DFG) Grant number Ni $130 / 11-1 / \mathrm{C}$ and formed part of G. Oberländer's diploma thesis.

\section{References}

Acott, T.S., Katz, D.F. \& Hoskins, D.D. (1983) Movement characteristics of bovine epididymal spermatozoa. Effects of forward motility protein and epididymal maturation. Biology of Reproduction 29, 389-399.
Bedford, J.M. (1990) Sperm dynamics in the epididymis. In Gamete Physiology, pp. 53-67. Eds R. A. Asch, J. P. Balmaceda \& I. Johnston. Serono Symposia, Norwell.

Billups, K.L., Tillman, S.L. \& Chang, T.S.K. (1990) Reduction of epididymal sperm motility after ablation 
of the inferior mesenteric plexus in the rat. Fertility and Sterility 53, 1076-1082.

Blandau, R.J. \& Rumery, R.E. (1964) The relationship of swimming movements of epididymal spermatozoa to their fertilizing capacity. Fertility and Sterility 15, 571-579.

Blazak, V.F., Ernst, T.L. \& Stewart, B.E. (1985) Potential indicators of reproductive toxicity: testicular sperm production and epididymal sperm number, transit time, and motility in Fischer 344 rats. Fundamental and Applied Toxicology 5, 1097-1103.

Boyers, S.P., Davis, R.O. \& Katz, D.F. (1989) Automated semen analysis. In Current Problems in Obstetrics, Gynecology and Fertility, pp. 169-200. Ed. R. L. Barbieri. Year Book Medical Publishers Inc., Chicago.

Burgos, M.H. \& Tovar, E.S. (1974) Sperm motility in the rat epididymis. Fertility and Sterility 25, 985-991.

Cornwall, G.A., Smyth, T.B., Vindnigh, D., Harter, C., Robinson, J. \& Chang, T.S.K. (1986) Induction and enhancement of progressive motility in hamster caput epididymal spermatozoa. Biology of Reproduction 35, 1065-1074.

Cornwall, G.A., Vindich, D., Tillman, S. \& Chang, T.S.K. (1988) The effect of oxidation on the morphology of immature hamster epididymal spermatozoa induced to acquire motility in vitro. Biology of Reproduction 39, 141-155.

DeGeyter, C., Bals-Pratsch, M., Dören, M., Yeung, C.H., Grunert, J.H., Bordt, J., Schneider, H.P.G. \& Nieschlag, E. (1988) Human and bovine cervical mucus penetration as a test of sperm function for in vitro fertilization. Human Reproduction 3, 948-954.

Douglas-Hamilton, D.H., Boisseau, P. \& Nett, W. (1990) Sperm velocity and motion parameter. Hamilton Thorn Research Report no. 21. Hamilton Thorn Research, Beverly.

Dyson, A.L.M.B. \& Orgebin-Crist, M-C. (1973) Effect of hypophysectomy, castration and androgen replacement upon the fertilizing ability of rat epididymal spermatozoa. Endocrinology 93, 391-402.

Fray C.S., Hoffer, A.P. \& Fawcett, D.W. (1972) A reexamination of motility patterns of rat epididymal spermatozoa. Anatomical Record 173, 301-308.

Gaddum-Rosse, P. (1981) Some observations on sperm transport through the uterotubal junction of the rat. American Journal of Anatomy 160, 333-341.

Ginsburg, K.A. \& Armant, D.R. (1990) The influence of chamber characteristic on the reliability of sperm concentration and movement measurements obtained by manual and videomicrographic analysis. Fertility and Sterility 53, 882-887.

Hinton, B.T., Dott, H.M. \& Setchell, B.P. (1979) Measurement of the motility of rat spermatozoa collected by micropuncture from the testis and from different regions along the epididymis. Journal of Reproduction and Fertility 55, 167-172.

Hinton, B.T., Brooks, D.E., Dott, H.M. \& Setchell, B.P. (1981) Effects of carnitine and some related compounds on the motility of rat spermatozoa from the caput epididymidis. Journal of Reproduction and Fertility 61, 59-64.

Kann, M.L. \& Serres, C. (1980) Development and initiation of sperm motility in the hamster epididymis. Reproduction, Nutrition and Development 20, $1739-1749$.
Katz, D.F., Davis, R.O. \& Delandmeter, B.A. (1985) Real-time analysis of sperm motion using automated video image digitization. Computer Methods and Program in Biomedicine 21, 173-184.

Klinefelter, G.R., Laskey, J.W., Roberts, N.R., Slott, V. \& Suarez, J.D. (1990) Multiple effects of ethane dimethanesulfonate on the epididymis of adult rats. Toxicology and Applied Pharmacology 105, 271-287.

Klinefelter, G.R., Gray, L.E. \& Suarez, J.D. (1991) The method of sperm collection significantly influences sperm motion parameters following ethane dimethanesulphonate administration in the rat. Reproductive Toxicology 5, 39-44.

Knuth, U.A. \& Nieschlag, E. (1988) Comparison of computerized semen analysis and sperm motion characteristics with the conventional procedure in 322 patients. Fertility and Sterility 49, 881-885.

Knuth, U.A., Yeung, C.H. \& Nieschlag, E. (1987) Computerized semen analysis: objective measurement of semen characteristics is biased by subjective parameter setting. Fertility and Sterility 48, 118-124.

Knuth, U.A., Yeung, C.H., Neuwinger, J. \& Nieschlang, E. (1989) Computerized semen analysis. In Recent Advances in Andrology, pp. 13-26. Eds M. Serio \& G. Waites. ARES-Serono Symposia, Rome.

Lee, W.M., Tsang, A.Y.F. \& Wong, P.Y.D. (1981) Effects of divalent and lanthanide ions on motility initiation in rat caudal epididymal spermatozoa. British Journal of Pharmacology 73, 633-638.

McClain, R.M. \& Downing, J.C. (1988) The effect of ornidazole on fertility and epididymal sperm function in rats. Toxicology and Applied Pharmacology 92, $488-496$.

Mack, S.O., Wolf, D.P. \& Tash, J.S. (1988) Quantitation of specific parameters of motility in large numbers of human sperm by digital image processing. Biology of Reproduction 38, 270-281.

Meizel, S., Lui, C.W., Working, P.K. \& Mrsny, R.J. (1980) Taurine and hypotaurine: their effects on motility, capacitation and the acrosome reaction of hamster sperm in vitro and their presence in sperm and reproductive tract fluids of several mammals. Development Growth and Differentiation 22, 483-494.

Mohri, H. \& Yanagimachi, R. (1980) Characteristics of motor apparatus in testicular, epididymal and ejaculated spermatozoa. Experimental Cell Research 127, 191-196.

Mortimer, D., Serres, C., Mortimer, S.T. \& Jouannet, P. (1988) Influence of image sampling frequency on the perceived movement characteristics of progressively motile human spermatozoa. Gamete Research 20, 313-327.

Neuwinger, J., Behre, H.M. \& Nieschlag, E. (1990) Computerized semen analysis with sperm tail detection. Human Reproduction 5, 719-723.

Olds-Clarke, P., Baer, H.M. \& Gerber, W.L. (1990) Human sperm motion analysis by automatic (Hamilton-Thorn motility analyzer) and manual (Image-80) digitization systems. Journal of Andrology $11,52-58$.

Pholpramool, C., Lea, O.A., Burrow, P.V., Dott, H.M. \& Setchell, B.P. (1983) The effects of acidic epididymal glycoprotein (AEG) and some other proteins on the motility of rat epididymal spermatozoa. International Journal of Andrology 6, 240-248. 
Rikmenspoel, R. (1984) Movements and active moments of bull sperm flagella as a function of temperature and viscosity. Journal of Experimental Biology 108, 205-230.

Serres, C. \& Kann, M.L. (1984) Motility induction in hamster spermatozoa from caput epididymidis: effects of forward motility protein (FMP) and calmodulin inhibitor. Reproduction, Nutrition Développement 24, 81-89.

Slott, V.L., Suarez, J.D., Simmons, J.E. \& Perreault, S.D. (1990) Acute inhalation exposure to epichlorohydrin transiently decreases rat sperm velocity. Fundamental and Applied Toxicology 15, 597-606.

Toth, G.P., Stober, J.A., Read, E.J., Zenick, H. \& Smith, M.K. (1989) The automated analysis of rat sperm motility following subchronic epichlorohydrin administration: methodologic and statistical considerations. Journal of Andrology 10, 401-415.

Toth, G.P., Stober, J.A., Zenick, H., Reads, E.J., Christ, S.A. \& Smith, M.K. (1991) Correlation of sperm motion parameters with fertility in rats treated subchronically with epichlorhydrin. Journal of Andrology 12, 54-61.

Turner, T.T. \& Giles, R.D. (1981) The effects of carnitine, glycerylphosphorylcholine, caffeine and egg yolk on the motility of rat epididymal spermatozoa. Gamete Research 4, 283-295.
Vantman, D., Koukoulis, G., Dennison, L., Zinamana, $M$ \& Sherins, R.J. (1988) Computer-assisted semen analysis: evaluation of method and assessment of the influence of sperm concentration on linear velocity. Fertility and Sterility 49, 510-515.

Woolley D.M. (1977) Evidence for 'twisted plane' undulations in golden hamster sperm tails. Journal of Cell Biology 75, 851-865.

Working, P.K. \& Hurtt, M.E. (1987) Computerized videomicrographic analysis of rat sperm motility. Journal of Andrology 8, 330-337.

Wyker, R. \& Howards, S.S. (1977) Micropuncture studies of the motility of rete testis and epididymal spermatozoa. Fertility and Sterility 28, 108-112.

Yeung, C.H. (1984) Effects of cyclic AMP on the motility of mature and immature hamster epididymal spermatozoa studied by reactivation of demembranated cells. Gamete Research 9, 99-114.

Yeung, C.H., Weinbauer, G.F. \& Nieschlag, E. (1989) Movement characteristics of ejaculated sperm from Cynomolgus monkeys (Macaca fascicularis) analyzed by manual and automated computerised image analysis. Journal of Medical Primatology 18, 55-68.

Received 15 July 1991 